\title{
CONFERENCE SCENE
}

For reprint orders, please contact: reprints@futuremedicine.com

\section{Movement disorders in Australia and beyond: a report from the Movement Disorder Society of Australia 2011 conference}

\author{
Alexander Münchau*
}

\section{The Movement Disorder Society of Australia 2011 conference, Melbourne, Australia, 8-10 September 2011}

The Movement Disorder Society of Australia 2011 conference was held in Melbourne, Australia, for 2 days in September to review recent advances in the field and provide an update on controversial issues. In the many oral presentations, poster session and video session the broad clinical spectrum of movement disorders was covered and directions of current and future research in the field summed up. Discourses and discussions on recent results of basic (neurobiological) science, systems neuroscience and clinical problems allowed participants to obtain a comprehensive overview of the diverse and growing subspecialty of movement disorders.

The Movement Disorder Society of Australia assembled for 2 days to comprehensively review recent advances in the field and highlight controversial issues. A number of relevant topics were discussed in a rigorous yet pleasant manner by both national and international experts and young researchers.

As the first invited key note speaker, Christopher Goetz (Rush University Medical Center, IL, USA), covered the challenging clinical problem of dyskinesias in Parkinson's disease (PD). He elegantly disentangled the sometimes confusing clinical phenomenology and emphasized the need for reliably rating dyskinesias with a particular view to patients' perception and quality of life, which is reflected in the development of the Movement Disorder Unified Dyskinesia Rating Scale; a reliable and valid tool that is currently translated into many languages other than English. In spite of an improved clinical description, classification and rating of dyskinesias and some advances in the understanding of their occurrence in the course of the disease as a consequence of striatal denervation, dopamine transporter downregulation and imbalances in nondopaminergic pathways including histamine $\mathrm{H} 3$ and cannabinoid receptors, Goetz made it clear that management is still unsatisfactory. As a medical treatment the only drugs with proven efficacy are amantadine and, to a lesser extent, clozapine. Deep-brain stimulation (DBS) of the subthalamic nucleus and the globus pallidus internus are surgical options but the use of amantadine/clozapine and DBS is limited by untoward side effects, particularly in the elderly. Goetz also discussed the conundrum that the duration of dyskinesias is typically increased
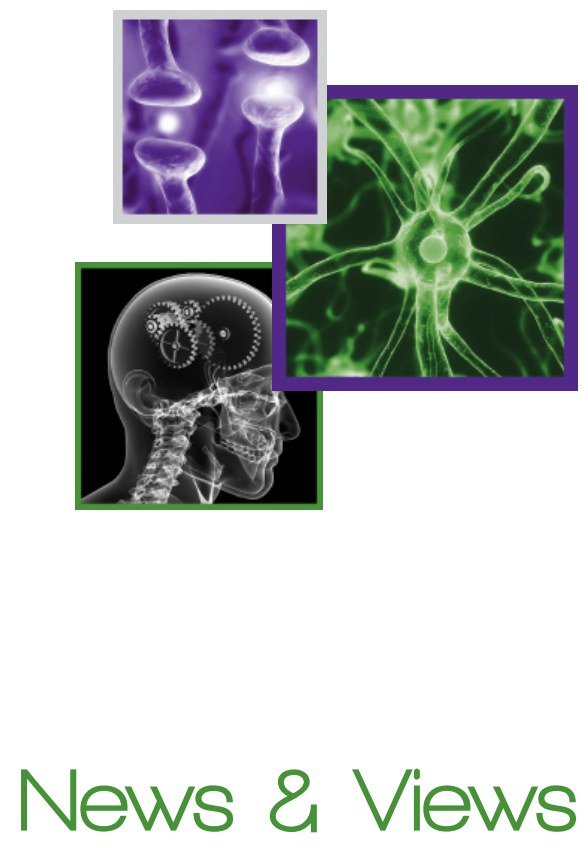

News

Journal Watch

Ask the Experts

Conference Scene

*Department of Neurology, University Hospital Medical Center Hamburg Eppendorf, Martinistrasse 52, 20246 Hamburg, Germany; Tel.: +49 407410 50134; Fax: +49 407410 55086; muenchau@uke.de 
by dosage increases of the dopaminergic medication but decreased, often by $50 \%$, by placebos which in turn also improve bradykinesia through the release of dopamine. Therefore, with regards to the antidyskinetic effect of placebos, other nondopaminergic neurotransmitter systems are likely to be involved.

Results of the ADAGIO trial, where a novel delayed-start design was used were discussed by Rick Stell from the Australian Neuromuscular Research Institute (Cottesloe, Australia). The trial suggested that rasagiline, an inhibitor of monoamine oxidase type $b$ that is approved for the symptomatic treatment of PD, might also have neuroprotective effects, however, only in PD patients taking $1 \mathrm{mg} /$ day but not in those taking $2 \mathrm{mg} /$ day. He particularly focussed on a subgroup analysis of the $2 \mathrm{mg}$ group which showed similar presumably neuroprotective effects in patients with higher clinical scores possibly indicating that in the group as a whole clinical severity was too mild for neuroprotective effects to become evident. He also evaluated the STRIDE-PD study where initiation of dopaminergic treatment with a combination of L-dopa/carbidopa/entacapone aiming at more continuous L-dopa availability compared with standard L-dopa/carbidopa treatment, unexpectedly failed to delay the time of onset or reduce the frequency of dyskinesia but was in fact associated with a shorter time to onset and increased frequency of dyskinesia. Stell pointed out that these disappointing results may have been due to long dosage intervals of $3.5 \mathrm{~h}$ rather than $3 \mathrm{~h}$. This important clinical study is another example that it can be difficult to prove that continuous nonpulsatile dopaminergic stimulation shown to prevent dyskinesias in animal models is also benefical in the clinical setting.

In my talks on phenomenology, pathogenesis, pathophysiology and management of Gilles de la Tourette syndrome, I reviewed recent studies supporting the view that etiologically genetic susceptibility plays a major role with some environmental factor (e.g., streptococcal infections) being possible disease modifiers. Given that neurophysiological and imaging studies have shown abnormalities in many different brain regions, there is no comprehensive pathophysiology model of tics or other symptoms and signs of this common childhood neuropsychiatric disorder. Recently, functional and structural abnormalities in several motor areas including the supplementary motor cortex, abnormal structural/functional relations in these areas and deficits of sensorimotor integration have come into focus underscoring the possible role of neurodevelopmental problems and aberrant reorganization. There have been no breakthroughs in pharmacological treatment but DBS of intralaminar nuclei of the thalamus and the globus pallidus internus has become an option in medically refractory cases with severe tics. However, evidence of short- and long-term efficacy of DBS is still very limited. Thus, it is recommended that DBS is carried out only in centers with both neuropsychiatric expertise in the management of Tourette syndrome and long standing experience with stereotactic neurosurgery, ideally in the setting of randomized controlled trials. Results of a recent positive clinical trial on habit reversal therapy to suppress or 'divert' unpleasant or bothersome tics later spurred a lively discussion on whether or not tic suppression is desirable, particularly in children given that reinforcement of inhibitory control may induce long-term 'entrainment' of a vicious circuit of tic inhibition followed by later tic 'surge'. As an alternative strategy some favored a more permissive attitude (free ticcing) and measures aiming primarily at educating and counseling the public through outreach activities to increase public awareness and reduce social stigma.

Carolyn Sue from Kolling Institute of Medical Research (St Leonards, Australia), introduced exciting human in vivo models of genetic movement disorders. For instance, on the basis of olfactory cell cultures gained from nasal biopsies of patients with Kufor-Rakeb syndrome, also known as PARK 9, a rare form of autosomal recessive parkinsonism associated with mutations in ATP13A2 (coding for a lysosomal type 5 P-type ATPase) they could demonstrate in vivo mislocalization of the mutant $A T P 13 A 2$, resultant stress of the endoplasmic reticulum, alterations in the proteasomal pathways and premature degradation of mutant ATP13A2 mRNA. These alterations likely contribute to the complex etiology of PARK 9. The concise and instructive talk by Carolyn Sue brought out the relevance of patientderived cell models to study the molecular pathophysiology of genetically determined movement disorders.

In his talk on immunologically mediated movement disorders Russell Dale, Westmead Hospital (Sydney, Australia), gave an overview of the increasingly recognized role of the immune system in the generation of some movement disorders. In Sydenham's chorea, a neuropsychiatric disorder associated with abnormal movements, inattention and impulsivity, the pathogenetic role of antistreptococcal antibodies cross reacting with basal ganglia antigens is well established. Whether or not antibodies play a role in pediatric autoimmune neuropsychiatric disorder associated with streptococcal infection (PANDAS) and whether such a syndrome exists at all remains controversial. On the other hand, the crucial role autoantibodies against NMDA receptors play in a considerable proportion of pediatric and adult patients with limbic encephalitis has become clear over the last 3 years. Patients with these NMDR receptor antibodies typically present with neuropsychiatric symptoms including acute or subacute psychosis, memory problems, frontal behavioral abnormalities, movement disorders, particularly chorea and unusual mouth and facial movements and autonomic symptoms. Some patients have a combination of symptoms reminiscent of what has been described as dyskinetic encephalitis lethargica, such as chorea, agitation, seizures and insomnia. Brain imaging in patients with NMDA receptors can be normal but CSF cell count is typically increased and oligoclonal bands are positive. Importantly, early diagnosis and aggressive treatment with steroids, immunoglobulins and rituximab can dramatically improve the outcome. It is very likely that other autoimmune syndromes presenting with movement disorders and neuropsychiatric syndromes will emerge in the future. 
As a keynote speaker, Sarah Tabrizi, Institute of Neurology (London, UK), comprehensively covered the challenging topic of Huntington's disease (HD) lookalikes comprising approximately $1 \%$ of patients with a clinical syndrome typical for HD. Most relevant diseases to consider are neuroacanthocytosis, McLeod syndrome, familial prion disease (HD-like disease 1), HD-like disease 2 caused by mutations in the $\mathrm{JPH} 3$ gene, the spinocerebellar ataxias type $1,2,3$ and 17 , dentate-rubro-pallido-lysian atrophy and disorders associated with abnormal brain iron accumulation. However, as Tabrizi stressed, most cases $(>95 \%)$ of HD-like diseases remain unclear. In spite of clinical similarities of these disorders underlying pathology and pathogenesis are manifold. Interestingly, however, in a recent study of a transgenic mouse model of HD-like 2 disease, caused by CTG/CAG repeat expansion at the JPH3 locus, molecular analyses revealed that a CAG repeat transcript encoded an expanded polyglutamine protein, which accumulated in a pattern quite similar to HD. The results suggest overlapping polyglutamine-mediated pathogenic mechanisms in HD and HD-like disease 2 , linking these disorders both at a clinical and molecular level.

The conference closed with an interesting video session bringing together basic scientists and clinicians. Through exchange of thoughts, ideas, acumen and discussions on different approaches to fathom clinical phenomenology; the session gave credit to the art of clinical problem solving in the field of movement disorders and neurology in general.

\section{Financial \& competing}

\section{interests disclosure}

Commercial research support grants from Pharm Allergan, Ipsen and Merz Pharmaceuticals. Honoraria for lectures from Pharm Allergan, Ipsen and Merz Pharmaceuticals. Support from a nonprofit foundation or society: Dystonia Medical Research Foundation (USA), Tourette syndrome Association (Germany) and N.E.MO. Charity (Germany) supporting the research of pediatric movement disorders. Academic research support not attributed in the manuscript: Deutsche Forschungsgemeinschaft (MU1692/2-2). A Münchau is employed at the University Hospital Medical Center Hamburg Eppendorf. The author has no other relevant affiliations or financial involvement with any organization or entity with a financial interest in or financial conflict with the subject matter or materials discussed in the manuscript apart from those disclosed.

No writing assistance was utilized in the production of this manuscript. 